\title{
ChemComm
}

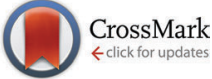

Cite this: Chem. Commun., 2016, 52,3231

Received 7th January 2016

Accepted 19th January 2016

DOI: $10.1039 / \mathrm{c} 6 \mathrm{cc} 00158 \mathrm{k}$

www.rsc.org/chemcomm

\section{Hydration-dependent anomalous thermal expansion behaviour in a coordination polymer $\dagger$}

\author{
Prem Lama, Lukman O. Alimi, Raj Kumar Das and Leonard J. Barbour*
}

\begin{abstract}
A coordination polymer is shown to possess anomalous anisotropic thermal expansion. Guest water molecules present in the as-synthesised material can be removed upon activation without loss of crystal singularity. The fully dehydrated form shows considerably different thermal expansion behaviour as compared to the hydrate.
\end{abstract}

Owing to longitudinal vibrational motion most materials expand along all three directions upon heating. This is known as positive thermal expansion (PTE). ${ }^{1}$ However, in isolated cases the occurrence of other low-energy molecular motions such as rigid unit modes (RUM), ${ }^{2}$ the invar effect ${ }^{3}$ etc. result in unusual thermal responses such as negative thermal expansion (NTE) ${ }^{4}$ or zero thermal expansion (ZTE). ${ }^{5} \mathrm{~A}$ few geometrically flexible materials have been reported to possess anisotropic thermal expansion as a result of novel types of mechanical responses that include hinge-like ${ }^{6}$ or stretching-tilting ${ }^{7}$ motion. A handful of organic and organic-inorganic hybrid materials such as $\mathrm{Ag}_{3}\left[\mathrm{Co}(\mathrm{CN})_{6}\right]$, Prussian blue analogues and methanol monohydrate have been reported to possess extraordinarily anisotropic thermal expansion. ${ }^{8}$ Being somewhat related to the abovementioned materials, MOFs have attracted substantial attention owing to their novel topologies and various potential applications. ${ }^{9}$ Additionally, isotropic negative thermal expansion has been reported for several MOF materials, including HKUST- $1,{ }^{10}$ IRMOFs $^{11}$ and cyano-bridged MOFs. ${ }^{12}$

Additional attention has recently been devoted to designing new materials that exhibit anomalous thermal expansion behaviour. However, as yet not much effort has been made to obtain tuneable thermal expansion, with the exception of a few reports of systems that display this highly desirable property. ${ }^{6 b, 13-16}$ The phenomenon of tuneable thermal expansion essentially involves invoking changes in weaker interactions or low frequency vibrational modes using external stimuli. For example, Chupas et al. have reported that NTE

Department of Chemistry and Polymer Science, University of Stellenbosch, Matieland, 7602, Stellenbosch, South Africa. E-mail: ljb@sun.ac.za

$\dagger$ Electronic supplementary information (ESI) available: Synthetic procedure, DSC analysis, detailed crystallographic information and additional figures. CCDC 1441452-1441470. For ESI and crystallographic data in CIF or other electronic format see DOI: $10.1039 / \mathrm{c} 6 \mathrm{cc} 00158 \mathrm{k}$ of $\mathrm{Zn}(\mathrm{CN})_{2}$ can be enhanced by the application of mechanical pressure to the system. ${ }^{13}$ The flexibility of the system can also be changed using chemical modification either pre $^{-14}$ or postsynthetically. ${ }^{6 b, 15,16}$ In the pre-synthetic approach, isostructural materials with different compositions can be designed, whereas the post-synthetic approach involves modification of framework flexibility either by changing the occupancy or nature of included guests in porous materials.

Herein, we report the modified synthesis of a coordination polymer $\left\{[\mathrm{Zn}(\mathrm{BTC})(\mathrm{HBPP})] \cdot \mathrm{H}_{2} \mathrm{O}\right\}_{n}\left(\mathbf{1} \cdot \mathrm{H}_{2} \mathrm{O}\right)$ (Scheme $\mathrm{S} 1$, ESI $\dagger$ ), which was previously prepared using a different procedure $(\mathrm{BTC}=1,3,5$-benzenetricarboxylate and $\mathrm{HBPP}=$ protonated 1,3-bis(4-pyridyl)propane). ${ }^{17}$ Single-crystal X-ray diffraction (SCD) analysis at $100 \mathrm{~K}$ reveals that $\mathbf{1} \cdot \mathrm{H}_{2} \mathrm{O}$ crystallises in the noncentrosymmetric orthorhombic space group $P n a 2_{1}$. The asymmetric unit consists of one $\mathrm{Zn}^{2+}$ ion, one $\mathrm{BTC}^{3-}$ ligand, one $\mathrm{HBPP}^{+}$ligand and a water molecule (Fig. S1, ESI $\dagger$ ). The $\mathrm{Zn}^{2+}$ ion adopts a distorted tetrahedral coordination geometry with three carboxylate groups bound to the metal centre, each in a monodentate coordination mode, and the fourth position is occupied by the pyridyl unit of the HBPP ligand. The remaining pyridyl unit of HBPP is protonated and therefore does not coordinate. The dihedral angles among the BTC units coordinated to the same $\mathrm{Zn}^{2+}$ centres are $12.8(1)^{\circ}, 34.2(1)^{\circ}$ and 36.7(1) (Fig. S2, ESI $\dagger$ ).

Two carboxylate groups of the BTC linkers bridge the metal centers to form approximately hexagonal helices along the crystallographic $b$ axis (Fig. 1). The pitch and diameter of the helices are 7.804(1) $\AA$ and $11.262(3) \AA$, respectively. The metal-metal distances along each helical chain are in the range 8.170(1) to 10.631(1) A. The individual helical columns are filled by the $\mathrm{HBPP}^{+}$ units and the protonated pyridyl groups are almost parallel to the BTC ligands, (Fig. 1) although there are no significant interactions between them. Each helical column is connected to neighbouring columns via carboxylate groups to generate an overall three dimensional structure (Fig. S3, ESI $\dagger$ ). Owing to the presence of both right- and left-handed helices, the framework is achiral (Fig. 2).

The overall structure contains small isolated (zero dimensional) voids that are occupied by the water molecules. The protonated 


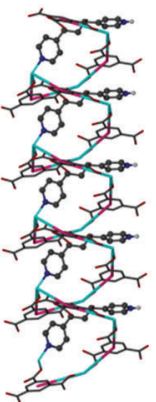

(a)

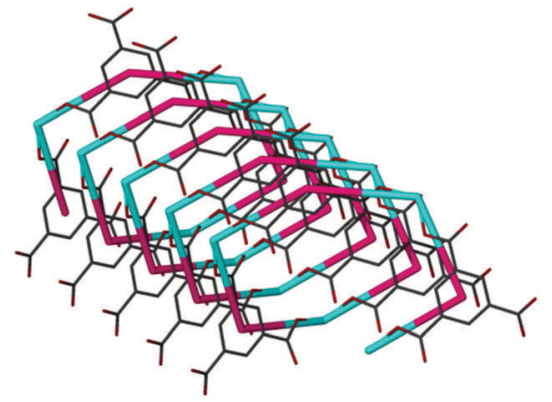

(b)
Fig. 1 (a) View of an approximately hexagonal helical chain with encapsulated $\mathrm{BPPH}^{+}$units and (b) perspective view of the helical chain approximately along [010] BPPH $^{+}$units are shown as ball-and-stick models while the remaining components are shown as wire and capped-stick models. All hydrogen atoms except those bonded to nitrogen atoms have been omitted for clarity).

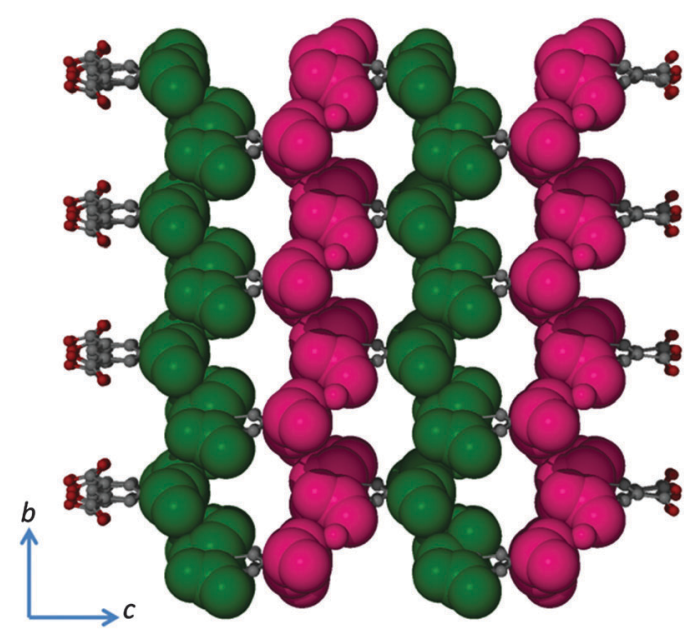

Fig. 2 Perspective view of right- and left-handed (pink and green, respectively) helical chains in $1 \cdot \mathrm{H}_{2} \mathrm{O}$ (hydrogen atoms and water molecules have been omitted and each helical column is shown as a space filled model).

pyridyl units and the water molecules form several $\mathrm{N}-\mathrm{H} \cdots \mathrm{O}$, $\mathrm{O}-\mathrm{H} \cdots \mathrm{O}$ and $\mathrm{C}-\mathrm{H} \cdots \mathrm{O}$ hydrogen bonding interactions (Fig. 3). Interestingly, all of the protonated pyridyl units are oriented in same direction (Fig. 3), consistent with the absence of inversion symmetry of the framework, and the coordination polymer is therefore polar.

The $\mathrm{Zn}^{2+}$ centre forms a pyramidal 3-connected node whereas the BTC units represent 3-connected planar nodes. The topology can thus be described as a 3-connected (10,3)-d (utp) net with extended Schläfli symbol $10_{2} \cdot 10_{4} \cdot 10_{4}$ (Fig. S4, ESI $\dagger$ ).

Variable-temperature single-crystal X-ray diffraction (VT-SCD) experiments were carried out with a view to understanding the temperature-dependent structural changes of $\mathbf{1} \cdot \mathrm{H}_{2} \mathrm{O}$. Starting at $260 \mathrm{~K}$, the crystal was cooled to $100 \mathrm{~K}$ and data were collected at $20 \mathrm{~K}$ intervals. Upon cooling the crystallographic $b$ axis contracts significantly while the $a$ and $c$ axes elongate gradually (Fig. 4). The linear thermal expansion coefficients along the three crystallographic axes $a, b$ and $c$ are $-6.0(1), 53.0(7)$ and

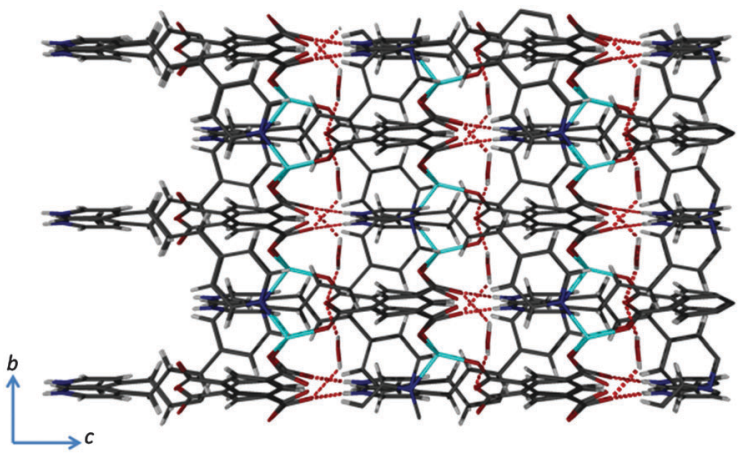

Fig. 3 View of the overall packing arrangement of $\mathbf{1} \cdot \mathrm{H}_{2} \mathrm{O}$ showing the $\mathrm{N}-\mathrm{H} \ldots \mathrm{O}$ and $\mathrm{O}-\mathrm{H} \ldots \mathrm{O}$ hydrogen bonding interactions (analogous $\mathrm{C}-\mathrm{H}$... O interactions are omitted for clarity).

$-7.9(5) \mathrm{MK}^{-1}$ respectively. Thus $\mathbf{1} \cdot \mathrm{H}_{2} \mathrm{O}$ shows rare biaxial negative thermal expansion behaviour. ${ }^{8 a, b, 16 b, 18}$ The overall volumetric thermal expansion is $39.0(8) \mathrm{MK}^{-1}$ (Fig. S5, ESI $\dagger$ ).

The reversibility of the thermal expansion process was verified by first cooling the crystal from $260 \mathrm{~K}$ to $100 \mathrm{~K}$ and then heating it again to $260 \mathrm{~K}$ (260K-R in ESI $\dagger)$. The initial and final unit cell dimensions at $260 \mathrm{~K}$ agree within experimental error.

In order to understand the mechanism responsible for the anomalous thermal expansion properties of $1 \cdot \mathrm{H}_{2} \mathrm{O}$, we analysed the types of hydrogen bonding interactions and temperaturedependent structural changes. The protonated pyridyl unit of the $\mathrm{HBPP}^{+}$present in each helical chain forms hydrogen bonding interactions with the carboxylate groups of the neighbouring helices. All of the $\mathrm{N}-\mathrm{H} \cdots \mathrm{O}$ hydrogen bonding interactions occur almost exclusively along the crystallographic $c$ axis (Fig. S6, ESI $\dagger$ ). Upon cooling, the flexible $\mathrm{HBPP}^{+}$linkers undergo conformational changes; the bond angle $\mathrm{C} 15 \cdots \mathrm{C} 16 \cdots \mathrm{C} 17\left(\theta_{1}\right)$ and the nonbonding torsion angle $\mathrm{N} 1 \cdots \mathrm{C} 12 \cdots \mathrm{C} 18 \cdots \mathrm{N} 2\left(\varphi_{1}\right)$ decrease from

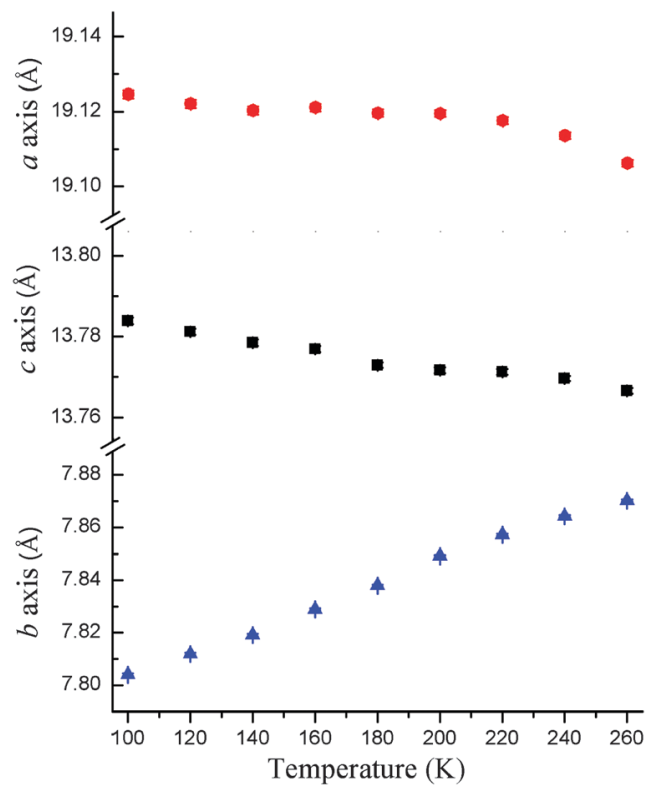

Fig. 4 Variation of unit cell dimensions of $1 \cdot \mathrm{H}_{2} \mathrm{O}$ (including error bars) with temperature. 
$111.5(3)^{\circ}$ to $110.7(2)^{\circ}$ and $7.4(5)^{\circ}$ to $6.3(4)^{\circ}$, respectively (Table S5, $\mathrm{ESI} \dagger)$. Thus the $\mathrm{HBPP}^{+}$linkers become more bent upon cooling, causing concomitant movement of BTC units and thus resulting in hinge-like motion of helical columns. Hence, the pitch of the helical columns formed by the BTC units decreases from 7.870(1) to 7.804(1) $\AA$ (Table S6, ESI $\dagger$ ) and the diameter of the helices increases from 11.239(3) to 11.262(3) A (Table S6, ESI $\dagger$ ) thereby resulting in PTE along the $b$ direction and NTE along the c direction.

The water molecules also bridge the carboxylate units between two neighbouring helical chains, forming several quite strong $\mathrm{O}-\mathrm{H} \cdots \mathrm{O}$ and weak $\mathrm{C}-\mathrm{H} \cdots \mathrm{O}$ hydrogen bonding interactions (Fig. S7, ESI $\dagger$ ). In this case these interactions act predominantly in the $a b$ plane (Fig. S7, ESI $\dagger$ ), thereby transferring a component of the hinge-like motion to the $a$ axis, and consequently causing NTE along this direction too. The $\mathrm{N}-\mathrm{H} \cdots \mathrm{O}$ and $\mathrm{O}-\mathrm{H} \cdots \mathrm{O}$ hydrogen bonding interactions in $\mathbf{1} \cdot \mathrm{H}_{2} \mathrm{O}$ are sufficiently strong to preclude any substantial temperature dependent changes in their characteristic geometrical parameters (Tables S7 and S8, ESI $\dagger$ ).

Thermogravimetric analysis (TGA) of $\mathbf{1} \cdot \mathrm{H}_{2} \mathrm{O}$ shows about $3.5 \%$ weight loss within the temperature range of 50 to $140{ }^{\circ} \mathrm{C}$, which corresponds to the loss of guest water molecules (calc. $3.7 \%$ ). The dehydrated framework appears to be stable up to $340{ }^{\circ} \mathrm{C}$ (Fig. S8, ESI $\dagger$ ). Based on the TGA results, crystals of $\mathbf{1} \cdot \mathrm{H}_{2} \mathrm{O}$ were activated at $140{ }^{\circ} \mathrm{C}$ under dynamic vacuum for 12 hours in order to remove the water guest molecules. Interestingly, the activated crystals retained their crystal singularity and SCD analysis at $100 \mathrm{~K}$ yielded the structure of the dehydrated framework 1. TGA of $\mathbf{1}$ also confirmed the absence of any residual water molecules (Fig. S8, ESI $\dagger$ ).

The framework of $\mathbf{1}$ is isoskeletal ${ }^{19}$ to that of its hydrated analogue. The $\mathrm{N}-\mathrm{H} \cdots \mathrm{O}$ hydrogen bonding interactions remain intact although, of course, the analogous $\mathrm{O}-\mathrm{H} \cdots \mathrm{O}$ interactions are absent in $\mathbf{1}$ owing to the loss of the guest water molecules. The dihedral angles between the BTC units change to $11.8(2)^{\circ}$, $38.3(2)^{\circ}$ and $40.2(2)^{\circ}$ owing to a slight structural rearrangement upon dehydration.

In order to elucidate the effect of dehydration on the flexibility of the framework, we also investigated the temperature-dependent structural changes in 1 . In this case the crystallographic $a$ axis remains practically constant in the temperature range 100 to $310 \mathrm{~K}$, whereas the thermal responses with respect to the crystallographic $b$ and $c$ axes remain quite similar to those of 1. $\mathrm{H}_{2} \mathrm{O}$ (Fig. 5). The linear thermal expansion coefficients along the crystallographic $a, b$ and $c$ directions are $-0.20(7), 47.8(7)$ and -7.4(7) $\mathrm{MK}^{-1}$, respectively. The dehydrated framework 1 therefore shows a very rare combination of positive, negative and zero thermal expansion behaviour. ${ }^{6,7}$ In this case, the overall volumetric thermal expansion is $40(1) \mathrm{MK}^{-1}$ (Fig. S9, ESI $\dagger$ ).

The thermal response of the BPP unit of $\mathbf{1}$ is somewhat different from that of the hydrated analogue; the non-bonding torsional angle $\mathrm{N} 1 \cdots \mathrm{C} 12 \cdots \mathrm{C} 18 \cdots \mathrm{N} 2\left(\varphi_{2}\right)$ increases from $7.5(8)^{\circ}$ to $13.0(5)^{\circ}$ whereas the bonding angle $\mathrm{C} 15 \cdots \mathrm{C} 16 \cdots \mathrm{C} 17\left(\theta_{2}\right)$ now decreases from $112.5(5)^{\circ}$ to $109.2(3)^{\circ}$ (Table S9, ESI $\dagger$ ). The BPP units bend upon cooling, the pitch of the helices decreases

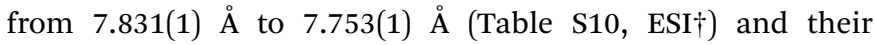

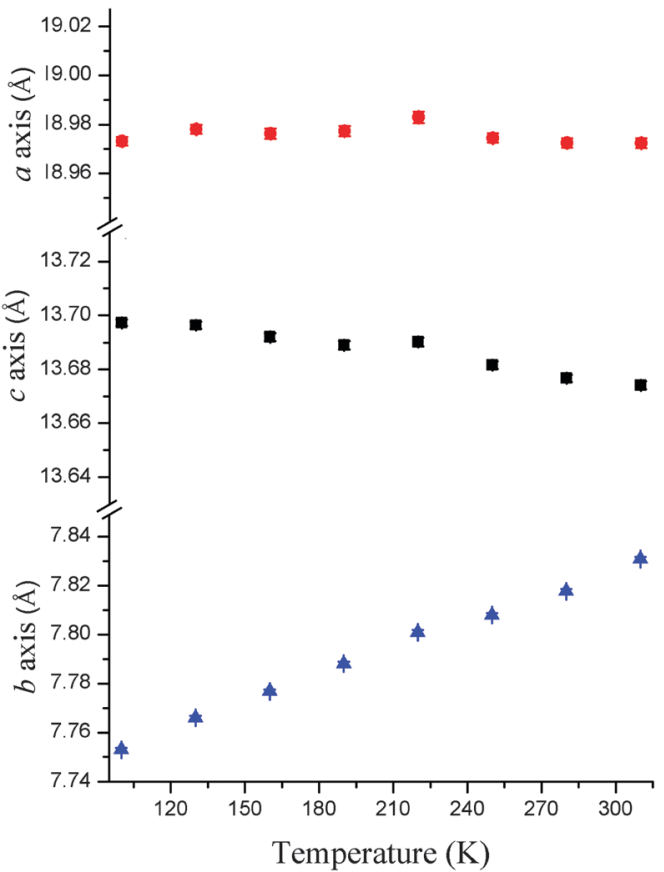

Fig. 5 Variation of unit cell dimensions of 1 (including error bars) with temperature.

diameter increases from 11.105(5) Å to 11.146(4) Å (Table S10, ESI $\dagger$ ). This results in similar thermal responses along the $b$ and $c$ directions as compared to the hydrated analogue. However, owing to the absence of $\mathrm{O}-\mathrm{H} \cdots \mathrm{O}$ and $\mathrm{C}-\mathrm{H} \cdots \mathrm{O}$ hydrogen bonding interactions in the dehydrated framework, the hinge-like motion cannot be transferred to the crystallographic $a$ axis, thereby resulting in zero thermal expansion along that direction. In this case, the $\mathrm{N}-\mathrm{H} \cdots \mathrm{O}$ hydrogen bonding interaction remains practically unaffected by temperature variation (Table S11, ESI $\dagger$ ). Differential scanning calorimetry (DSC) of both the hydrated and the dehydrated frameworks rules out any possibility of a temperatureinduced phase transition - i.e. no distinct thermal events were observed during the thermal cycle in either case (Fig. S10, ESI $\dagger$ ).

To date, guest-dependent thermal expansion behaviour has been reported mostly for materials that display NTE purely due to transverse vibrational motion. ${ }^{10,15}$ For $\mathrm{Cd}(\mathrm{CN}) 2,{ }^{15 a} \mathrm{ZnPt}(\mathrm{CN})_{6}{ }^{15 b}$ and HKUST- $1^{10}$ negative thermal expansion behaviour was observed in the guest-free frameworks whereas quite ordinary PTE was observed in the guest-filled systems. In MOF-5, the NTE of the host framework becomes less pronounced under gas pressure as compared to its evacuated form. ${ }^{15 c}$ In these instances, PTE of the included guest molecules either dampens or compensates for the NTE of the host framework. The presence of empty channels is necessary for these materials to undergo prominent NTE.

To date there have been very few reports of the thermal expansion behaviour of a host being enhanced upon guest uptake. $^{6 b, 16}$ In FMOF-1 the NTE of the host framework is enhanced by $\mathrm{N}_{2}$ pressure ${ }^{16 a}$ but gas-loading in the system gradually increases upon cooling, leading to guest-induced phase transitions, as indicated by the two-step breathing process. To the best of 
our knowledge it is extremely rare to observe guest-induced enhancement of a negative thermal response where the guest occupancy remains constant throughout the thermal cycle. ${ }^{6 b, 16 b}$ We have previously shown how the thermal response of the MOF $\left[\mathrm{Zn}(\mathrm{L})_{2}(\mathrm{OH})_{2}\right]_{n} \quad(\mathrm{~L}=4$ - $(1 H$-naphtho[2,3- $d]$ imidazole-1yl)benzoic acid) increases upon inclusion of primary alcohols $(\mathrm{MeOH}, \mathrm{EtOH}$ and $n \mathrm{PrOH})$ but diminishes with inclusion of iPrOH. ${ }^{16 b}$ However, such guest dependent behaviour has been attributed to the change in framework flexibility upon desolvation and exchange. Since the guest molecules other than those hydrogen bonded to the $[\mathrm{Zn}-\mathrm{OH}-]_{n}$ chain could not be modelled, the influence of the guest on the flexibility is not understood. Chen et al. recently reported a similar type of guest-induced enhancement of thermal expansion behaviour ${ }^{6 b}$ but in their case the guest molecules were also highly disordered. In the present report the position of the guest water molecule in $\mathbf{1} \cdot \mathrm{H}_{2} \mathrm{O}$ has been located precisely and the influence of its non-covalent interactions can therefore be rationalised. Consequently, the changes in the weaker interactions and framework dynamics resulting from desolvation can be clearly understood. However, in this case the water molecules occupy a small proportion of the volume and thus the changes in the overall packing arrangement upon dehydration are also very small. Consequently, the changes in the overall thermal response are relatively insignificant compared to those reported by Saha et al. ${ }^{16 c}$

The preparation of porous materials such as $\mathbf{1}$, together with a detailed elucidation of the guest-dependent changes in their packing arrangements and host-guest interactions may help to establish the design principles for materials having controllable thermal expansion behaviour. Such exploratory studies could lead to several potential single-crystal devices such as thermomechanical sensors or actuators.

We are grateful to the National Research Foundation and the Department of Science and Technology (SARCHI Program) for financial support of this work. PL also acknowledges the Claude Leon Foundation for financial support.

\section{Notes and references}

1 N. W. Ashcroft and N. D. Mermin, Solid State Physics, Holt, Rinehart \& Winston, 1976.

2 (a) W. Miller, C. W. Smith, D. S. Mackenzie and K. E. Evans, J. Mater. Sci., 2009, 44, 5441-5451; (b) C. Lind, Materials, 2012, 5, 1125-1154.

3 (a) C. E. Guillaume, C. R. Acad. Sci., 1897, 125, 235-238; (b) C. E. Guillaume, Nature, 1904, 71, 134-139; (c) M. van Schilfgaarde, I. A. Abrikosov and B. Johansson, Nature, 1999, 400, 46-49.

4 (a) V. K. Peterson, G. J. Kearly, Y. Wu, A. J. Ramirez-Cuesta, E. Kemner and C. J. Kepert, Angew. Chem., Int. Ed., 2010, 49, 585-588; (b) A. L. Goodwin and C. J. Kepert, Phys. Rev. B: Condens. Matter Mater. Phys., 2005, 71, 140301.

5 (a) A. E. Phillips, G. J. Halder, K. W. Chapman, A. L. Goodwin and C. J. Kepert, J. Am. Chem. Soc., 2010, 132, 10-11; (b) S. Margadonna, K. Prassides and A. N. Fitch, J. Am. Chem. Soc., 2004, 126, 15390-15391; (c) X. Song, Z. Sun, Q. Huang, M. Rettenmayr, X. Liu, M. Seyring, G. Li, G. Rao and F. Yin, Adv. Mater., 2011, 23, 4690-4694; (d) Y.-S. Wei, M. Zhang, P.-Q. Liao, R.-B. Lin, T.-Y. Li, G. Shao, J.-P. Zhang and X.-M. Chen, Nat. Commun., 2015, 6, 8348.
6 (a) S. Henke, A. Schneemann and R. A. Fischer, Adv. Funct. Mater., 2013, 23, 5990-5996; (b) H.-L. Zhou, R.-B. Lin, C.-T. He, Y.-B. Zhang, N. Feng, Q. Wang, F. Deng, J.-P. Zhang and X.-M. Chen, Nat. Commun., 2013, 4, 2534; (c) R. K. Das, H. Aggarwal and L. J. Barbour, Inorg. Chem., 2015, 54, 8171-8173.

7 P. Lama, R. K. Das, V. J. Smith and L. J. Barbour, Chem. Commun., 2014, 50, 6464-6467.

8 (a) D. Das, T. Jacobs and L. J. Barbour, Nat. Mater., 2010, 9, 36-39; (b) D. Das, T. Jacobs, A. Pietraszko and L. J. Barbour, Chem. Commun., 2011, 47, 6009-6011; (c) A. D. Fortes, E. Suard and K. S. Knight, Science, 2011, 331, 742-746; (d) A. L. Goodwin, M. Calleja, M. J. Conterio, M. T. Dove, J. S. O. Evans, D. A. Keen, L. Peters and M. G. Tucker, Science, 2008, 319, 794-797; (e) K. M. Hutchins, R. H. Groeneman, E. W. Reinheimer, D. C. Swenson and L. R. MacGillivray, Chem. Sci., 2015, 6, 4717-4722; $(f)$ H.-L. Zhou, Y.-B. Zhang, J.-P. Zhang and X.-M. Chen, Nat. Commun., 2015, 6, 6917.

9 (a) R. Banerjee, H. Furukawa, D. Britt, C. Knobler, M. O'Keeffe and O. M. Yaghi, J. Am. Chem. Soc., 2009, 131, 3875-3877; (b) J. J. Perry IV, J. A. Perman and M. J. Zaworotko, Chem. Soc. Rev., 2009, 38, 1400-1417; (c) J. M. Ogborn, I. E. Collings, S. A. Moggach, A. L. Thompson and A. L. Goodwin, Chem. Sci., 2012, 3, 3011-3017; (d) R. K. Das, A. Aijaz, M. K. Sharma, P. Lama and P. K. Braradwaj, Chem. - Eur. J., 2012, 18, 6866-6872; (e) M. Eddaoudi, D. F. Sava, J. F. Eubank, K. Adil and V. Guillerm, Chem. Soc. Rev., 2015, 44, 228-249; $(f)$ S. Sen, S. Neogi, K. Rissanen and P. K. Bharadwaj, Chem. Commun., 2015, 51, 3173-3176.

10 (a) V. K. Peterson, G. J. Kearly, Y. Wu, A. J. Ramirez-Cuesta, E. Kemner and C. J. Kepert, Angew. Chem., Int. Ed., 2010, 49, 585-588; (b) Y. Wu, A. Kobayashi, G. J. Halder, V. K. Peterson, K. W. Chapman, N. Lock, P. D. Southon and C. J. Kepert, Angew. Chem., Int. Ed., 2008, 120, 9061-9064.

11 (a) N. Lock, Y. Wu, M. Christensen, L. J. Cameron, V. K. Peterson, A. J. Bridgeman, C. J. Kepert and B. B. Iverson, J. Phys. Chem. C, 2010, 114, 16181-16186; (b) D. Dubbeldam, K. S. Walton, D. E. Ellis and R. Q. Snurr, Angew. Chem., Int. Ed., 2007, 46, 4496-4499.

12 (a) A. L. Goodwin and C. J. Kepert, Phys. Rev. B: Condens. Matter Mater. Phys., 2005, 71, 140301; (b) J. L. Korcok, M. L. Katz and D. L. Lenznott, J. Am. Chem. Soc., 2009, 131, 4866-4871.

13 K. W. Chapman and P. J. Chupas, J. Am. Chem. Soc., 2007, 129, 10090-10091.

14 (a) A. L. Goodwin, D. A. Keen, M. G. Tuker, M. T. Love. L. Peters and J. S. O. Evans, J. Am. Chem. Soc., 2008, 130, 9660-9661; (b) S. G. Duyker, V. K. Peterson, G. J. Kearley, A. J. Ramirez-Cuesta and C. J. Kepert, Angew. Chem., Int. Ed., 2013, 52, 5266-5270; (c) K. Oka, K. Nabetani, C. Sakaguchi, H. Seki, M. Czapski, Y. Shimakawa and M. Azuma, Appl. Phys. Lett., 2013, 103, 061909; (d) S. Bhattacharya, V. G. Saraswatula and B. K. Saha, Cryst. Growth Des., 2013, 13, 3651-3656; (e) K. W. Chapman, P. J. Chupas and C. J. Kepert, J. Am. Chem. Soc., 2006, 128, 7009-7014.

15 (a) A. E. Phillips, A. L. Goodwin, G. J. Halder, P. D. Southon and C. J. Kepert, Angew. Chem., Int. Ed., 2008, 47, 1396-1399; (b) A. L. Goodwin, K. W. Chapman and C. J. Kepert, J. Am. Chem. Soc., 2005, 127, 17980-17981; (c) N. Lock, M. Christensen, C. J. Kepert and B. B. Iversen, Chem. Commun., 2013, 49, 789-791.

16 (a) C. Yang, X. Wang and M. A. Omary, Angew. Chem., Int. Ed., 2009, 48, 2500-2505; (b) I. Grobler, V. J. Smith, P. M. Bhatt, S. A. Herbert and L. J. Barbour, J. Am. Chem. Soc., 2013, 135, 6411-6414; (c) V. G. Saraswatula and B. K. Saha, Cryst. Growth Des., 2015, 15, 593-601.

17 J. Zhang, Y.-B. Chen, S.-M. Chen, Z.-J. Li, J.-K. Cheng and Y.-G. Yao, Inorg. Chem., 2006, 45, 3161-3163.

18 (a) S. J. Hibble, A. M. Chippindale, A. H. Pohl and A. C. Hannon, Angew. Chem., Int. Ed., 2007, 46, 7116-7118; (b) L. Zhang, X. Kuang, X. Wu, W. Yang and C. Lu, Dalton Trans., 2014, 43, 7146-7152; (c) Y.-S. Wei, K.-J. Chen, P.-Q. Liao, B.-Y. Zhu, R.-B. Lin, H.-L. Zhou, B.-Y. Wang, W. Xue, J.-P. Zhang and X.-M. Chen, Chem. Sci., 2013, 4, 1539-1546.

19 (a) G. O. Lloyd, J. Alen, M. W. Bredenkamp, E. J. C. de Vries, C. Esterhuysen and L. J. Barbour, Angew. Chem., Int. Ed., 2006, 45, 5354-5358; (b) J. L. Atwood, L. J. Barbour and A. Jerga, Science, 2002, 296, 2367-2369; (c) S. Bhattacharya and B. K. Saha, CrystEngComm, 2011, 13, 6941-6944. 